\title{
Collaborative Simulation Grid: \\ Multiscale Quantum-Mechanical/Classical Atomistic Simulations on Distributed PC Clusters in the US and Japan
}

\author{
Hideaki Kikuchi, "Rajiv K. Kalia,", Aiichiro Nakano,", Priva Vashishta ${ }^{*, \dagger}$ \\ ${ }^{\dagger}$ Computer Science Department, Department of Materials Science, \\ Department of Physics \& Astronomy, Department of Biomedical Engineering, \\ University of Southern Califomia, USA \\ *Concurrent Computing Laboratory for Materials Simulations, Department of Computer Science, \\ Department of Physics \& Astronomy, Louisiana State University, USA \\ hkikuchi@phys.lsu.edu, (anakano, kalia, priyav)@usc.edu \\ Hiroshi Iyetomi \\ Department of Physics, Niigata University, Japan \\ hiyetomi@sc.niigata-u.ac.jp \\ Shuji Ogata, Takahisa Kouno \\ Department of Applied Sciences, Yamaguchi University, Japan \\ ogata@yamaguchi-u.ac.jp \\ Fuyuki Shimojo \\ Faculty of Integrated Arts and Sciences, Hiroshima University, Japan \\ shimojo@minerva.ias.hiroshima-u.ac.jp \\ Kenji Tsuruta \\ Department of Electrical and Electronic Engineering, Okayama University, Japan \\ tsuruta@elec.okayama-u.ac.jp \\ Subhash Saini \\ IT Modeling and Simulation, NASA Ames Research Center, USA \\ saini@nas.nasa.gov
}

\begin{abstract}
A multidisciplinary, collaborative simulation has been performed on a Grid of geographicaily distributed PC clusters. The multiscale simulation approach seamlessly combines i) atomistic simulation based on the molecular dynamics (MD) method and ii) quantum mechanical (QM) calculation based on the density functional theory (DFT) so that accurate but less scalable computations are performed only where they are needed. The multiscale MD/QM simulation code has been Grid-enabled using i) a modular, additive hybridization scheme, ii) multiple QM clustering, and iii) computation/communication overlapping. The Gridified MD/QM simulation code has been used to study environmental effects of water molecules on fracture in silicon. A preliminary run of the code has achicved a parallel efficiency of $94 \%$ on 25 PCs distributed over 3 PC slusters in the US and Japan, and a larger test involving 154 processors on 5 distributed PC clusters is in progress.
\end{abstract}

Keywords: Grid application, multiscale simulation, molecular dynamics, quantum mechanics, density functional theory

$0-7695-1524-X 02 \$ 17.00$ (c) 2002 IEEE 


\section{Introduction}

Metacomputing on a Grid of geographically distributed computing platforms connected via high-speed networks could revolutionize computational research, by enabling collaborative, hybrid computations that integrate multiple expertise distributed over wide geographical locations [1]. The availability of inexpensive PC clusters at the research-group level suggests a new collaborative mode for computational research, in which multiple research groups of diverse expertise participate in a metacomputing project by providing both expert-maintained application programs and computational resources to run them. Such a multidisciplinary application is emerging at the forefront of computational sciences and engineering. The multiscale simulation embeds accurate quantum mechanical (QM) calculations to handle chemical reactions (below a length scale of $10^{-8} \mathrm{~m}$ ) within a molecular dynamics (MD) simulation to describe Iarge-scale atomistic processes (up to a length scale of $10^{-6} \mathrm{~m}$ ), see Fig. 1 [2-5]. Modern design of high-performance materials and devices focuses on controlling structures at diverse length scales from atomic to macroscopic [6], and such multiscale $\mathrm{MD} / \mathrm{QM}$ simulations are expected to play an important role in scaling down engineering concepts to nanometer scales.

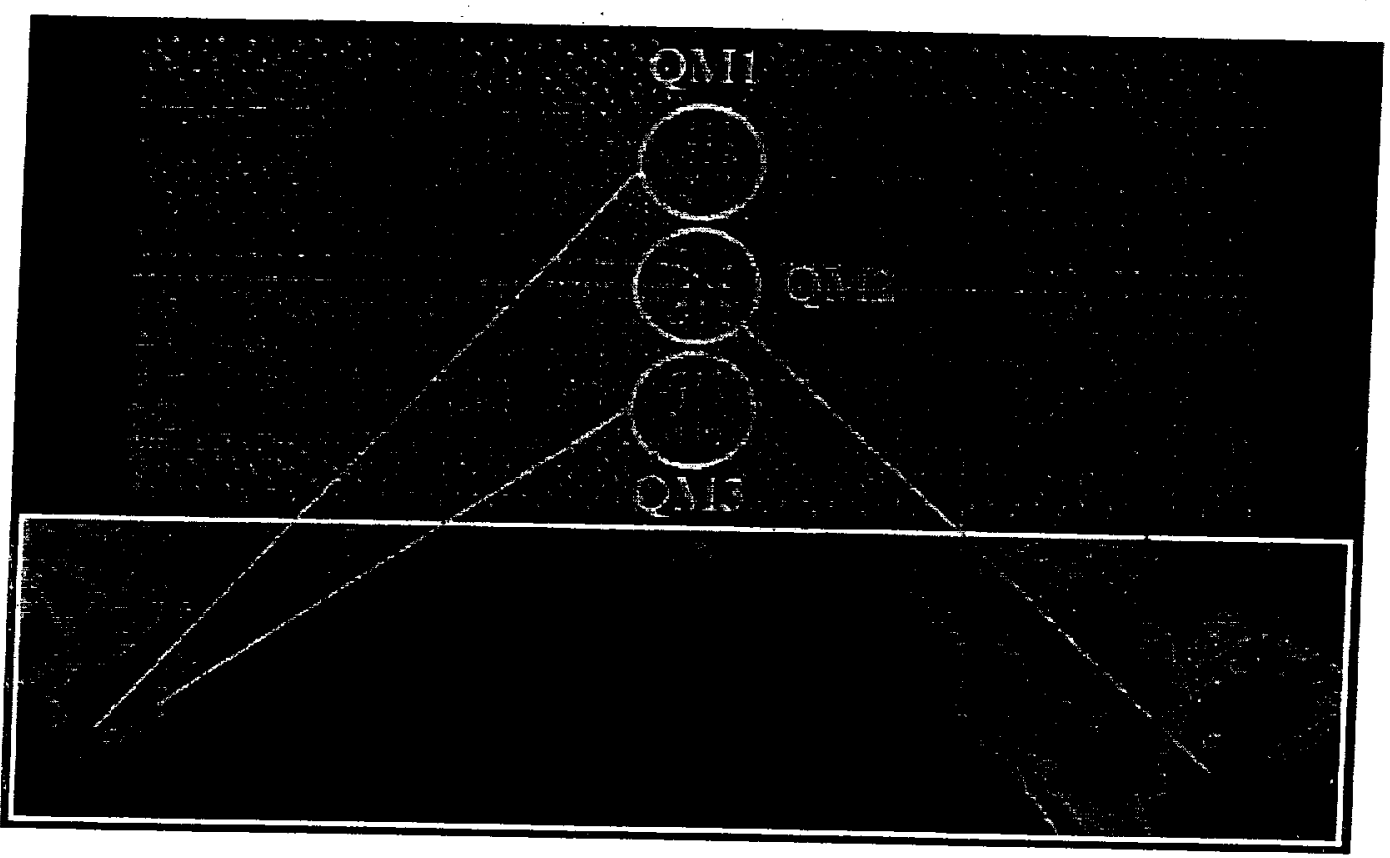

Figure 1: Multiscale MD/QM simulation of the reaction of water molecules at a crack tip in silicon (top), performed on geographically distributed PC clusters in the US and Japan (bottom). In this example, three QM calculations are embedded in an MD simulation, where green spheres represent QM silicon atoms; blue, handshake silicon atoms; red, QM oxygen atoms; yellow, QM hydrogen atoms; gray, MD silicon atoms.

Unfortunately, only a limited class of scientific and engineering applications has been shown to scale on such Grid computing platforms [7]. This paper describes our efforts, to enable MD/QM simulations on a Grid by designing scalable multiscale simulation algorithms. in the next section, we describe MD and QM algorithms as well as their Grid-enabled hybridization schemes. Section 3 discusses Grid implementation of the resulting MD/QM simulation algorithm. Results of benchmark tests are given in Sec. 4 , and Sec. 5 contains conclusions.

\section{Multiscale simulation algorithms}

We have developed a multiscale simulation algorithm that combines a classical MD simulation and a self-consistent QM calculation based on the density functional theory (DFT). 


\subsection{Space-time multiresolution molecular dynamics algorithm}

The MD approach obtains the phase-space trajectories (positions and velocities of all atoms at all time) by numerically integrating coupled ordinary differential equations. The dynamics is encoded in the interatomic potential energy, $E_{\mathrm{MD}}\left(\mathbf{r}^{N}\right)$, which is a function of the positions of all $N$ atoms, $\mathbf{r}^{N}=\left\{\mathbf{r}_{1}, \mathbf{r}_{2}, \ldots, \mathbf{r}_{i}\right\}$, in the system. In our many-body interatomic potential scheme, $E_{\mathrm{MD}}\left(\mathbf{r}^{N}\right)$ is an analytic function that depends on relative positions of atomic pairs and triples [3].

We have developed highly efficient, multiresolution algorithms to carry out large-scale MD simulations on parallel computers [8,9]. The most compute-intensive problem in an MD simulation is the $O\left(N^{2}\right)$ computation of the electrostatic energy for $N$ charged atoms. We use the Fast Multipole Method (FMM) [10] to reduce the complexity to $O(N)$ by computing the electrostatic field recursively on an octree. In our multiresolution molecular dynamics (MRMD) algorithm, the FMM is combined with a symplectic, multiple time-scale (MTS) method [11] that applies different force-updating schedules for different force components while keeping the phase-space volume occupied by the atoms as a simulation loop invariant.

Our MRMD program also features wavelet-based computational-space decomposition for adaptive load balancing [12,13] and spacefilling-curve-based adaptive data compression for scalable VO [14].

\subsection{Real-space quantum-mechanical calculation based on the density functional theory}

Empirical interatomic potentials used in MD simulations fail to describe chemical processes. Instead, interatomic interaction in reactive regions needs to be calculated by a QM method that can describe breaking and formation of bonds. An atom consists of a nucleus and surrounding electrons, and quantum mechanics explicitly treats the electronic degrees-of-freedom. Since each electron's wave function is a linear combination of multiple states, the combinatorial solution space for a many-electron problem is exponentially large. Walter Kohn received a Nobel prize in 1998 for the development of a heuristic called the density functional theory (DFT) [15-17]. The DFT avoids the exhaustive enumeration of many-electron correlations by solving $N_{\mathrm{wf}}$ single-electron problerns in a common average envirenment, which is determined self-consistently $\left(N_{w f}\right.$ is the number of independent wave functions or electronic bands). As a result, the problem is reduced to a self-consistent matrix eigenvalue problem, which can be solved with $O\left(N_{\mathrm{wi}}{ }^{3}\right)$ operations. The DFT problem can be formulated as the minimization of an energy functional, $E_{\mathrm{QM}}\left(\Psi^{N w f}\right)$, with respect to electron wave functions, $\psi^{\text {Nwf }}(\mathbf{r})=\left\{\psi_{1}(\mathbf{r}), \Psi_{2}(\mathbf{r}), \ldots, \psi_{N_{w f}}(r)\right\}$, subject to orthonormalization constraints on the wave functions.

- For efficient parallel implementation of the DFT, we employ real-space approaches based on higher-order finite differencing [18] and multigrid acceleration [19,20]. Our parallel DFT code $[9,21,22]$ includes electron-ion interactions using norm-conserving pseudopotentials [23] and the exchange-correlation energy in a generalized gradient approximation [24].

\subsection{Multiscale molecular-dynamics/quantum-mechanical simulation algorithm}

We have developed a multiscale $\mathrm{MD} / \mathrm{QM}$ simulation scheme, in which atomic clusters described by $\mathrm{QM}$ calculation are embedded in an atomistic region (see Fig. 1) [3-5]. The motion of atoms is described with a real-space multigrid-based DFT in the QM clusters and with the MD approach in the surrounding region. To make the $\mathrm{MD} / \mathrm{QM}$ simulation scalable on a Grid, we have used the following techniques.

Additive hybridization. To minimize the modification of existing $\mathrm{MD}$ and $\mathrm{QM}$ codes, we employ an additive hybridization approach based on a linear combination of MD and QM energies [25]. The total energy, $E$, is written as

$$
E=E_{\mathrm{MD}}^{\mathrm{yystem}}+E_{\mathrm{QM}}^{\text {cluster }}\left(\left\{\mathbf{r}_{\mathrm{QM}}\right\},\left\{\mathbf{r}_{\mathrm{HS}}\right\}\right)-E_{\mathrm{MD}}^{\text {cluster }}\left(\left\{\mathbf{r}_{\mathrm{QM}}\right\},\left\{\mathbf{r}_{\mathrm{HS}}\right\}\right),
$$

where $E_{\mathrm{MD}}^{\mathrm{bystem}}$ is the classical MD energy for the entire system and the last two terms represent the QM energy correction for the QMi cluster: $\varepsilon_{\mathrm{QM}}^{\text {cluster }}$ is the QMi energy for the cluster (its dangling bonus are terminated by hydrogen atoms to provide appropriate boundary conditions), and $E_{\mathrm{MD}}^{\mathrm{cluster}}$ is the MD potential energy of the cluster (terminated by appropriate $\mathrm{MD}$ atoms). In $\mathrm{Eq} .(1),\left\{\mathbf{r}_{\mathrm{QM}}\right\}$ is the set of the positions of the $\mathrm{QM}$ atorns, and $\left\{\mathbf{r}_{\mathrm{HS}}\right\}$ are the positions of handshake (HS) atoms, i.e., classical atoms bonded to the $\mathrm{QM}$ aroms (see Fig. 1). In $\mathrm{QM}$ calculation, each bond connecting a QM atom and a HS atom is terminated by a hydrogen atom. Positions of the termination hydrogen atoms are determined dynamically with the scaled-position link atom method as a function of 
$\left\{\mathbf{r}_{\mathrm{QM}}\right\}$ and $\left\{\mathrm{r}_{\mathrm{HS}}\right\}$. Other physical quantities, such as interatomic forces, are derived from Eq. (1) as a linear combination as well. Because of this additivity, our cut-and-paste type hybridization scheme is highly modular. these modules can easily be combine the MD and QM modules independently on their own PC clusters, and then is not trivial for most hybridization to a single metacomputing application on the Grid of their PC clusters. (This coordinates in the outer region.)

Equation (1) shows that, far from the MD/QM boundary, the MD and QM systems are characterized by the MD and $Q M$ energies, respectively. The additive $M D / Q M$ scheme linearly interpolates the two physical models at the a Si surface, we he and an such, its validity must be tested by the smoothess of the interpolation. For oxidation energy of an oxygen moleculated the time variation of the kinetic energy distribution to show that the dissociation $\mathrm{MD} / \mathrm{QM}$ boundary [4]

Multiple QM clustering. For scalable $\mathrm{MD} / \mathrm{QM}$ simulations on a Grid, we have implemented a divide-andconquer scheme, in which the QM region is a union of multiple QM clusters (see Fig. 1) [5]. Since the energy is a sum of the QM energy corrections for the clusters in the additive hybridization scheme,

$$
E=E_{\mathrm{MD}}^{\text {system }}+\sum_{\text {cluster }}\left[E_{\mathrm{QM}}^{\text {cluster }}\left(\left\{\mathbf{r}_{\mathrm{QM}}\right\},\left\{\mathbf{r}_{\mathrm{HS}}\right\}\right)-E_{\mathrm{MD}}^{\text {cluster }}\left(\left\{\mathbf{r}_{\mathrm{QM}}\right\},\left\{\mathbf{r}_{\mathrm{HS}}\right\}\right)\right] \text {, }
$$

each QM-cluster calculation does not access the atomic coordinates in the other clusters, and accordingly its paralle] computationally more efficient-QM-cluster communication. Furthermore, the multiple-QM-cluster scheme is prefactor of $O(N)$ DFT algorithms makes single-QM-cluster scheme because of the $O\left(N^{3}\right)$ scaling. (The large 22\}.)

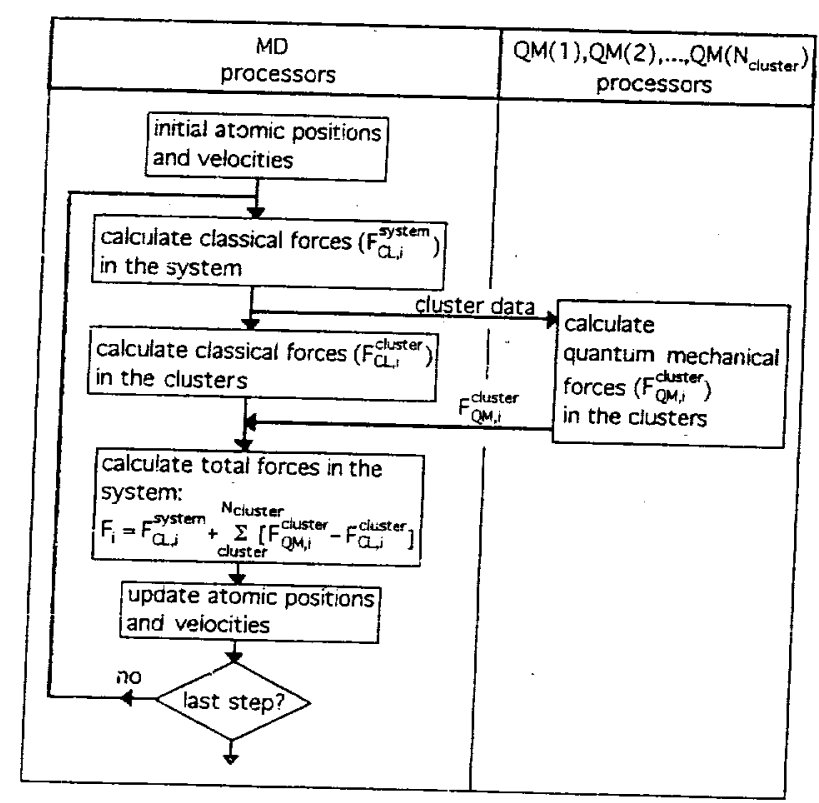

Figure 2: A flowchart of parallel computations in the hybrid $\mathrm{MD} / \mathrm{QM}$ simulation algorithm.

The hybrid $\mathrm{MD} / \mathrm{QM}$ simulation algorithm has been implemented on parallel computers, by first dividing processors into the $\mathrm{MD}$ and $\mathrm{QM}$ calculations (task decomposition) and then using spatial decomposition in each
task. The additive hybridizations task. The additive hybridization scheme makes the MD and $\mathrm{QM}$ subtasks entirely independent except for the compute the energy and forces of the entire system forces, as shown in the flowchart in Fig. 2. The MD processors the QM processor groups. Subsequently, the MD and $\mathrm{QM}$ processors indepentes of the QM clusters to each of computations on the atomic clusters. The QM energy and forces are then independently perform the $M D$ and $Q M$ 
total energy and corresponding forces are calculated and the equations of motion are integrated to update the atomic positions and velocities. The communications between the MD and QM processors are minimal, since the MD processors only need to send several hundred atomic coordinates to each QM cluster, which in retum sends back the calculated several hundred force components. (This is in contrast to non-additive hybridization schemes, in which the QM tasks continuously access the atomic information in the outer region during their computations.)

Computation/communication overlap. To hide the latency, the communications between the MD and QM processors have been overlapped with the computations. Our spatial decomposition scheme splits the computation on each processor into the interior and boundary computations. The interior computation is then fully overlapped with the communication of the boundary data.

\section{Grid implementation}

The above hybridization scheme is amenable to meta-computing on a Grid. We have implemented the multiscale $\mathrm{MD} / \mathrm{QM}$ simulation algorithm as a single MPI (Message Passing Interface) program. The Globus middleware (www.globus.org) and the Grid-enabled MPI implementation, MPICH-G2 (www3.niu.edu/mpi), have been used to implement the MPI-based multiscale MD/QM simulation code in a Grid environment.

In the parailel MD/QM program, all the tasks constitute a single MPI communicator, MPI_COMM_WORLD, and processors are grouped into MD and QM groups by defining multiple MPI communicators. (The MD calculation, as well as each of the QM-cluster calculations, is assigned a dedicated communicator.) The code is written in a single-program multiple-data (SPMD) style, which uses selection statements for the MD and QM processors to execute only the corresponding code segments. Dynamic memory allocation/deallocation operations in Fortran 90 are used to reduce the memory size.

In the current implementation, processors on multiple PC clustess are statically allocated using a host file. The user specifies the number of processors for each QM-cluster calculation in a configuration file. (On heterogeneous platforms, load balancing needs to be manually achieved by assigning more processors on slower platforms.) The additive hybridization and multiple QM clustering schemes minimize the inter-task communications, and thus the maximal parallel efficiency is achieved by assigning each QM cluster to a single PC cluster. Each PC cluster, on the other hand, can have multiple QM clusters without much communication overhead.

The QM calculation involves significant $\mathrm{I} / \mathrm{O}$ for saving wave functions of all the electrons. For example, the Qiv calculation of 76 atoms ( $36 \mathrm{Si}$ and $40 \mathrm{H}$ atoms) produces 24 MBytes of data. (The wave functions in the previous 2 MD steps are saved to obtain a good initial guess for the iterative solution by extrapolation.) To avoid the $\mathrm{V} / \mathrm{O}$ bottleneck, these wave functions are saved locally within each PC clister.

\section{Performance tests}

We have implemented the hybrid MD/QM simulation code on geographically distributed PC clusters in the US and Japan. The Linux-based PC clusters used for this project include:

- A 65-processor Intel Pentium IV 2.0GHz cluster at Yamaguchi University in Japan;

- Three 24-processor Intel Pentium IV $1.8 \mathrm{GHz}$ clusters at Hiroshima, Okayama, and Niigata Universities in Japan;

- A 17-processor AMD Athlon XP 1900+ cluster at the Louisiana State University (LSU) in the US.

These PCs are connected using Gigabit Ethemet switches inside each cluster and uplink to campus backbone. Nondedicated networks used for the Grid include the SINET/SuperSINET (OC192), APAN/TransPAC (OC12), Abilene (OC48), LaNet (OC3), and campus networks of the participating institutions. However, the measured bandwidth between LSU and the other institutions in Japan is low (ranging from 50 to $200 \mathrm{kBytes} / \mathrm{s}$ ) due to the bottleneck of the low-bandwidth campus networks and bandwidth sharing.

A preliminary benchmark test of the hybrid MD/QM simulation code has been performed on the Grid of PC clusters described above. (A larger benchmark test involving 154 processors is being performed on 5 PC clusters in the US and Japan.) $\mathrm{MD} / \mathrm{QM}$ simulations have been performed to study environmental effects of $\mathrm{H}_{2} \mathrm{O}$ molecules on fracture of strained $\mathrm{Si}$, in which atoms near the crack tip are treated quantum-mechanically in the framework of the DFT. Significant effects of the stress intensity factor on the reaction of the $\mathrm{H}_{2} \mathrm{O}$ molecules at the crack tip have been thereby observed [5]. The simulated system is a cracked Si with (110) crack surfaces under uniaxial tension, containing 91,256 atoms. The total number of $\mathrm{QM}$ atoms is $76 \mathrm{~N}_{\text {cluster }}$ ( $36 \mathrm{Si}$ and 40 termination-H atoms per cluster) 
where $N_{\text {cluster }}$ is the total number of QM clusters. At least several hundred QM atoms are needed to accurately mode]
the crack tip region.

Figure 3 shows the execution time of the $\mathrm{MD} / \mathrm{QM}$ simulation code as a function of $N_{\text {che }}$ where each cluster calculation is performed on an 8 -processor $\mathrm{PC}$ cluster. The total number of processors is thus $P=1+8 N_{\text {che }}$, (the $\mathrm{MD}$ simulation is performed on 1 processor). In the run on 25 processors, the PC cluster at LSU performs the MD task as well as 1 QM-cluster calculation on $9(=1+8)$ processors, whereas each of the PC clusters at Niigata and Yamaguchi uses 8 processors to perform 1 QM-cluster calculation.

Since the system size in the QM calculations scales linearly with the number of processors, the constant execution time per simulation step signifies perfect speedup. (As shown in Fig. 3, the execution time for the MD task is nearly constant, since the number of MD atoms is kept constant.) We first define the speed of the MD/QM program as a product of the total number of QM atoms and time steps executed in a second. The scaled speedup is given by the ratio between the speed of $N_{\text {cluster }}$ clusters and that of one cluster. The scaled efficiency is the scalec MDeedup divided by $N_{\text {cluster. }}$ On 25 processors ( 3 PC clusters) in the US and Japan, the measured executicn time per MD step is 224.8 seconds. This is only a slight increase from 211.2 seconds on 1 PC cluster in a single location, and the resulting fficiency is 0.94 .

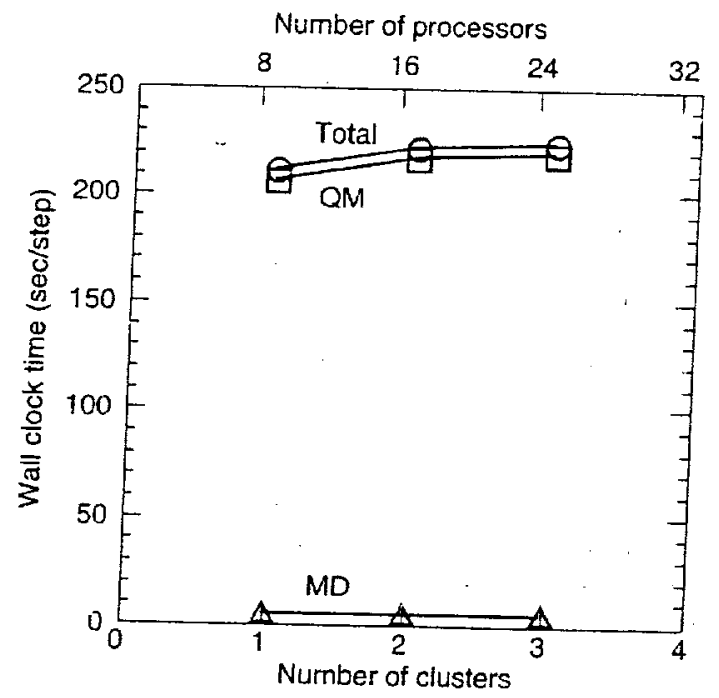

Figure 3: Wall clock time per simulation step for the MD/QM simulation code as a function of the number of $\mathrm{QM}$ clusters, $N_{\text {cluster. }}$ (The number of simulated QM atoms is $76 N_{\text {cluster. }}$ ) Each QM-cluster calculation is performed on an 8 -processor PC cluster, and the MD simulation is performed on 1 processor. The number of processors in the US

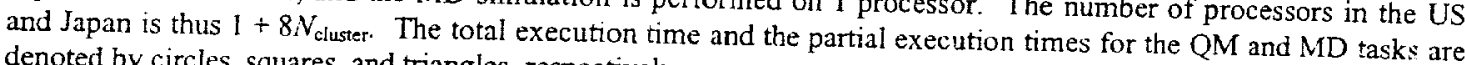
denoted by circles, squares, and triangles, respectively.

As is evidert in Fig. 3, the dominant computation in the MD/QM simulation is the QM task. Table I shows the averaged wall clock and communication times per MD step for three different stages of the QM task: i) Initial setup; ii) iterative solution of the eigenvalue problem; and iii) force calculation. Not only is the second stage computationally dominant, but it also involves considerable communication.

TABLE I: Partial wall clock and communication times per MD step for the QM task.

\begin{tabular}{|c|c|c|c|}
\hline & Initialization & Eigenvalue problem & Force calculation \\
\hline Wall clock time (sec) & 16.5 & 192.7 & 1.5 \\
\hline Communication $(\mathrm{sec})$ & 1.0 & 67.9 & 1.3 \\
\hline
\end{tabular}


The computation of the $\mathrm{QM}$ task scales as $O\left(N^{3} / P\right)$ for $N$ QM atoms on $P$ processors, whereas the communication scales as $O\left(N(N / P)^{2 / 3}+N^{2} \log P\right)$ [22]. The communication overhead becomes less for large grain sizes, $N / P$, but could become a bottleneck if a QM cluster is assigned on multiple PC clusters with weak communication links.

\section{Conclusions}

We have demonstrated the scalability of multiscale MD/QM simulations on a Grid of geographically distributed PC clusters in the US and Japan. The resulting high efficiency (despite the weak communication link) suggests that this is an efficient collaboration mode in computational research. Such a multiscale simulation collaboratory will consist of geographically distributed application specialists who not only maintain their computational modules with most updated simulation algorithms but also provide compute servers that are best suited to their own application codes. We have shown that the additive multiple-clustering scheme is effective in Gridifying such multiscale simulation codes. Furthermore, the additive hybridization scheme allows multiple layers of nested hybridization includirg, e.g., MD, DFT, and higher accuracy QM calculations such as configuration interaction. These methods are currently used by separate groups of scientists to solve similar problems at different levels of accuracy and problem sizes. The multiscale simulation collaboratory on a Grid will allow these scientists to jointly solve clallenging scientific/engineering problems. The Gridification approach in this paper should be applicable to other modular multiscale simulations as well. An example is the black-box hybridization scheme based on the multilevel Newton method, in which the residual equation is formulated in a coupled model space combining, e.g., electrostatic and mechanical problems [26].

\section{Acknowledgements}

The work at LSU was partially supported by AFOSR, ARL, DOE, NASA, NSF, USC-Berk:ley-Princeton-LSU DURINT, and Louisiana Board of Regents. Programs have been developed using parallel computers ar the NASA Armes Research Center and the i66-processor PC cluster at the Concurrent Computing Laboratory for Materials Simulations (CCLMS) at Louisiana State University. The work in Japan was supported by the Japan Science and Technology Corporation, Research and Deveiopment Program for Applying Advanced Computational Science and Technology

\section{References}

[1] I. Foster and C. Kesselman. The Grid: Blueprint for a New Computing Infrastructure. Morgan Kaufmann, San Francisco, 1999.

[2] F. F. Abraham, J. Q. Broughton, N. Bernstein, and E. Kaxiras. Spanning the length scales in dynamic simulation. Computational Physics, 12:538-546 (1998).

[3] A. Nakano, M. E. Bachlechner, R. K. Kalia, E. Lidorikis, P. Vashishta, G. Z. Voyiadjis, T. J. Campbell, S Ogata, and F. Shimojo. Multiscale simulation of nanosystems. Computing in Science and Engineering, $3(4): 56.66(2001)$.

[4] S. Ogata. E. Lidorikis, F. Shimojo, A. Nakano, P. Vashishta, and R. K. Kalia. Hybrid finite-elementimoleculardynamics'electronic-density-functional approach to materials simulations on parallel computers. Computer Physics Communications, 138:143-154 (2001).

[5] S. Ogata, F. Shimojo, A. Nakano, P. Vashishta, and R. K. Kalia. Environment effects of $\mathrm{H}_{2} \mathrm{O}$ on fracture initiation in Si: a hybrid electronic-density-functional/molecular-dynamics study. Journal of Applied Physics, submitted (2002).

[6] A. Pechenik, R. K. Kalia, and P. Vashishta. Computer-Aided Design of High-Temperature Materials. Cxford Univ. Press, Oxford, 1999. 
[7] G. Allen, T. Dramlitsch, I. Foster, N. T. Karonis, M. Ripeanu, E. Seidel, B. Toonen. Supporting efficient execution in heterogeneous distributed computing environments with Cactus and Globus. In Proceedings of
Supercomputing 2001, ACM, New York, 2001.

[8] A. Nakano, R. K. Kalia, and P. Vashishta. Multiresolution molecular dynamics algorithm for realistic materials modeling on parallel computers. Computer Physics Communications, 83:197-214 (1994).

[9] A. Nakano, R. K. Kalia, P. Vashishta, T. J. Campbell, S. Ogata, F. Shimojo, and S. Saini. Scalable atomistic simulation algorithms for materials research. In Proceedings of Supercomputing 2001, ACM, New York, 2001. [10] L. Greengard and V. Rokhlin. A fast algerithm for particle simulations. Journal of Computational Physics,
$73: 325-348$ (1987).

[11] M. E. Tuckerman, D. A. Yarne, S. O. Samuelson, A. L. Hughes, and G. J. Martyna. Exploiting multiple levels of parallelism in molecular dynamics based calculations via modern techniques and software paradigms on distributed memory computers. Computer Physics Communications, 128:333-376 (21)00). [12] A. Nakano. Multiresolution load balancing in curved space: The wavetet representation. Cuncurrency:
Practice and Experience, 11:343-353 (1999).

[13] A. Nakino and T. J. Campbell. An adaptive clrvilinear-coordinate approach to dynamic load balancing of paralle! multi-resolution molecular dynamics, Parallel Computing, 23:1461-1478 (1997).

[14] A. Omeltchenko, T. J. Campbell, R. K. Kalia, X. Liu, A. Nakano, and P. Vashishta. Scalable I/O of large-scale
molecular-dynamics simulations: A data-compression algorithm. Computer Physics Communicutions, 131.78 85 (2000).

[15] P. Hohenberg arıd W. Kohn. Inhomogeneous electron gas. Physical Review, 136:B864-B871 (1964).

[16] W. Kohn and P. Vashishta. General density functional theory. In Inhomogeneous Electron Gas, eds. N. H. March and S. Lundqvist, pages 79-184. Plenum, New York, 1983.

[17] M. C. Payne, M. P. Teter, D. C. Allan, T. A. Arias, and I. D. Joannopoulos. Iterative minimization techniques for $a \dot{b}$ initio total energy calculations: molecular dynarnics and conjugate gradients. Reviews of Modern Physics, 64:1045-1097 (1992).

[18]J. R. Chelikowsky, Y. Saad, S. Ögüt, I. Vasiliev, and A. Stathopoulos. Electronic structure methods for predicting the properties of materials: Grids in space. Phyica Status Solidi (b), 217:173-195 (2000),

$[19]$ T. L. Beck. Real-space mesh techniques in density-functional thəory. Reviews of Modern Physics, 72:1041.
1080 (2000).

$[20] \mathrm{J}$-L. Fattebert and J. Bernholc. Towards grid-based $O(N)$ density-functional theory methods: Optimized nonorthogonal orbitals and multigrid acceleration. Physical Review B, 62:1713-1722 (2000).

[21] F. Shimojo, T. J. Campbell, R. K. Kalia, A. Nakano, P. Vashishta, S. Ogata, and K. Tsuruta. A scalable molecular-dynamics-algorithm suite for materials simulations: Design-space diagram on !,024 Cray T3E
processors. Future Generation Computer Systems, 17:279-291 (2000).

[22] F. Shimojo, R. K. Kalia, A. Nakano, and P. Vashishta. Linear-scaling density-functional-theory calculations of electronic structure based on real-space grids: Design, analysis, and scalability test of parallel algorithms. Computer Physics Communications, 140:303-314 (2001)

[23] N. Troullier and J. L. Martins. Efficient pseudopotentials for plane-wave calculations. Physical Review $B$,
43:1993-2006 (1991).

[24] J. P. Perdew, K. Burke, and M. Emzerhof. Generalized gradient approximation made simple. Physical Revien
Letters, 77:3865-3868 (1996).

[25] S. Dapprich, I. Kom romi, K. S. Byun, K. Morokuma, and M. J. Frisch. A new ONIOM implementation in Gaussian 98. I. The calculation of energies, gradients, vibrational frequencies, and electric field derivatives Journal of Molecular Structure (Theochem) 461-462:1-21 (1999).

[26]N. R. Aluru and J. White. A multilevel Newton method for mixed-energy domain simulation of MEMS Journal of Microelectromechanical Systems, 8:299-308 (1999). 